\title{
Anisotropic Triangulations via Discrete Riemannian Voronoi Diagrams ${ }^{* \dagger}$
}

\author{
Jean-Daniel Boissonnat ${ }^{1}$, Mael Rouxel-Labbé2 ${ }^{2}$, and \\ Mathijs Wintraecken ${ }^{3}$
}

1 INRIA Sophia Antipolis Méditerranée, Valbonne, France

2 INRIA Sophia Antipolis Méditerranée, Valbonne, France; and

GeometryFactory, Valbonne, France

3 INRIA Sophia Antipolis Méditerranée, Valbonne, France

\begin{abstract}
The construction of anisotropic triangulations is desirable for various applications, such as the numerical solving of partial differential equations and the representation of surfaces in graphics. To solve this notoriously difficult problem in a practical way, we introduce the discrete Riemannian Voronoi diagram, a discrete structure that approximates the Riemannian Voronoi diagram. This structure has been implemented and was shown to lead to good triangulations in $\mathbb{R}^{2}$ and on surfaces embedded in $\mathbb{R}^{3}$ as detailed in our experimental companion paper.

In this paper, we study theoretical aspects of our structure. Given a finite set of points $\mathcal{P}$ in a domain $\Omega$ equipped with a Riemannian metric, we compare the discrete Riemannian Voronoi diagram of $\mathcal{P}$ to its Riemannian Voronoi diagram. Both diagrams have dual structures called the discrete Riemannian Delaunay and the Riemannian Delaunay complex. We provide conditions that guarantee that these dual structures are identical. It then follows from previous results that the discrete Riemannian Delaunay complex can be embedded in $\Omega$ under sufficient conditions, leading to an anisotropic triangulation with curved simplices. Furthermore, we show that, under similar conditions, the simplices of this triangulation can be straightened.
\end{abstract}

1998 ACM Subject Classification Computational Geometry and Object Modeling

Keywords and phrases Riemannian Geometry, Voronoi diagram, Delaunay triangulation

Digital Object Identifier 10.4230/LIPIcs.SoCG.2017.19

\section{Introduction}

Anisotropic triangulations are triangulations whose elements are elongated along prescribed directions. Anisotropic triangulations are known to be well suited when solving PDE's [10, $19,24]$. They can also significantly enhance the accuracy of a surface representation if the anisotropy of the triangulation conforms to the curvature of the surface [15].

Many methods to generate anisotropic triangulations are based on the notion of Riemannian metric and create triangulations whose elements adapt locally to the size and anisotropy prescribed by the local geometry. The numerous theoretical and practical results [1] of the Euclidean Voronoi diagram and its dual structure, the Delaunay triangulation, have pushed authors to try and extend these well-established concepts to the anisotropic setting.

\footnotetext{
* A full version of the paper is available at https://arxiv.org/abs/1703.06487.

$\dagger$ The first and third authors have received funding from the European Research Council under the European Union's ERC Grant Agreement number 339025 GUDHI (Algorithmic Foundations of Geometric Understanding in Higher Dimensions).
} 

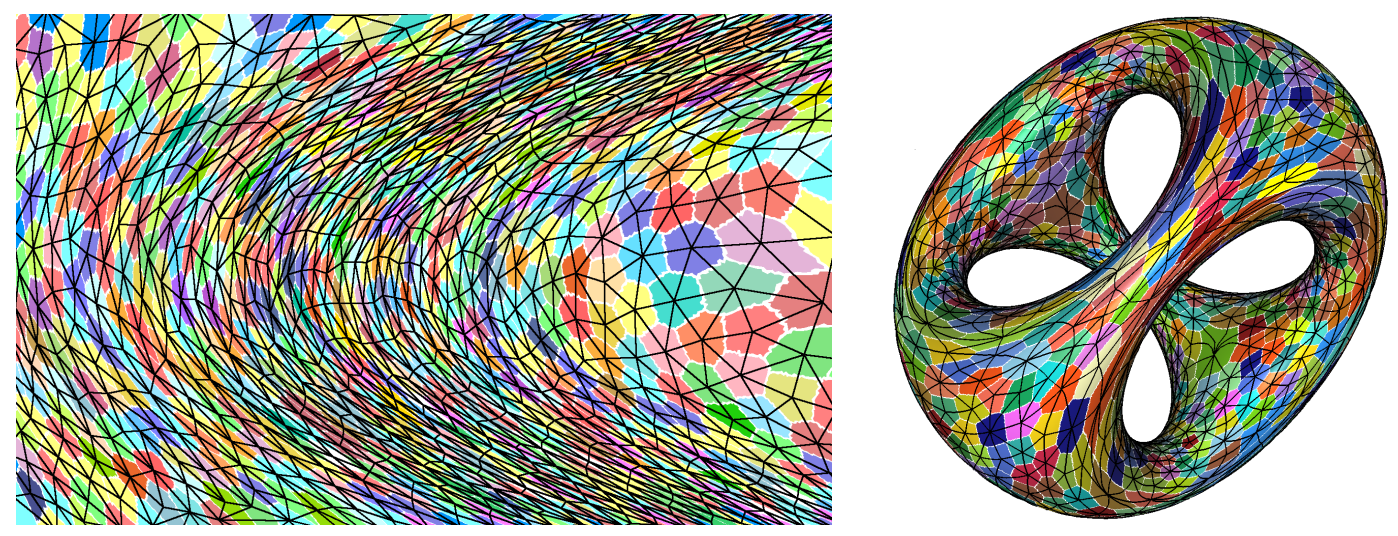

Figure 1 Left, the discrete Riemannian Voronoi diagram (colored cells with bisectors in white) and its dual complex (in black) realized with straight simplices of a two-dimensional domain endowed with a hyperbolic shock-based metric field. Right, the discrete Riemannian Voronoi diagram and the dual complex realized with curved simplices of the "chair" surface endowed with a curvature-based metric field [23].

Labelle and Shewchuk [17] and Du and Wang [12] independently introduced two anisotropic Voronoi diagrams whose anisotropic distances are based on a discrete approximation of the Riemannian metric field. Contrary to their Euclidean counterpart, the fact that the dual of these anisotropic Voronoi diagrams is an embedded triangulation is not immediate, and, despite their strong theoretical foundations, the anisotropic Voronoi diagrams of Labelle and Shewchuk and Du and Wang have only been proven to yield, under certain conditions, a good triangulation in a two-dimensional setting $[6,7,9,12,17]$.

Both these anisotropic Voronoi diagrams can be considered as an approximation of the exact Riemannian Voronoi diagram, whose cells are defined as $V_{g}\left(p_{i}\right)=\left\{x \in \Omega \mid d_{g}\left(p_{i}, x\right) \leq\right.$ $\left.d_{g}\left(p_{j}, x\right), \forall p_{j} \in \mathcal{P} \backslash p_{i}\right\}$, where $d_{g}(p, q)$ denotes the geodesic distance. Their main advantage is to ease the computation of the anisotropic diagrams. However, their theoretical and practical results are rather limited. The exact Riemannian Voronoi diagram comes with the benefit of providing a more favorable theoretical framework and recent works have provided sufficient conditions for a point set to be an embedded Riemannian Delaunay complex [2, 14, 18]. We approach the Riemannian Voronoi diagram and its dual Riemannian Delaunay complex with a focus on both practicality and theoretical robustness. We introduce the discrete Riemannian Voronoi diagram, a discrete approximation of the (exact) Riemannian Voronoi diagram. Experimental results, presented in our companion paper [23], have shown that this approach leads to good anisotropic triangulations for two-dimensional domains and surfaces, see Figure 1.

We introduce in this paper the theoretical side of this work, showing that our approach is theoretically sound in all dimensions. We prove that, under sufficient conditions, the discrete Riemannian Voronoi diagram has the same combinatorial structure as the (exact) Riemannian Voronoi diagram and that the dual discrete Riemannian Delaunay complex can be embedded as a triangulation of the point set, with either curved or straight simplices. Discrete Voronoi diagrams have been independently studied, although in a two-dimensional isotropic setting by Cao et al. [8]. 


\section{Riemannian geometry}

In the main part of the text we consider an (open) domain $\Omega$ in $\mathbb{R}^{n}$ endowed with a Riemannian metric $g$, which we shall discuss below. We assume that the metric $g$ is Lipschitz continuous. The structures of interest will be built from a finite set of points $\mathcal{P}$, which we call sites.

\subsection{Riemannian metric}

A Riemannian metric field $g$, defined over $\Omega$, associates a metric $g(p)=G_{p}$ to any point $p$ of the domain. This means that for any $v, w \in \mathbb{R}^{n}$ we associate an inner product $\langle v, w\rangle_{g}=$ $v^{t} g(p) w$, in a way that smoothly depends on $p$. Using a Riemannian metric, we can associate lengths to curves and define the geodesic distance $d_{g}$ as the minimizer of the lengths of all curves between two points. When the map $g: p \mapsto G$ is constant, the metric field is said to be uniform. In this case, the distance between two points $x$ and $y$ in $\Omega$ is $d_{G}(x, y)=\|x-y\|_{G}=\sqrt{(x-y)^{t} G(x-y)}$.

Most traditional geometrical objects can be generalized using the geodesic distance. For example, the geodesic (closed) ball centered on $p \in \Omega$ and of radius $r$ is given by $B_{g}(p, r)=\left\{x \in \Omega \mid d_{g}(p, x) \leq r\right\}$. In the following, we assume that $\Omega \subset \mathbb{R}^{n}$ is endowed with a Lipschitz continuous metric field $g$.

We define the metric distortion between two distance functions $d_{g}(x, y)$ and $d_{g^{\prime}}(x, y)$ to be the function $\psi\left(g, g^{\prime}\right)$ such that for all $x, y$ in a small-enough neighborhood we have: $1 / \psi\left(g, g^{\prime}\right) d_{g}(x, y) \leq d_{g^{\prime}}(x, y) \leq \psi\left(g, g^{\prime}\right) d_{g}(x, y)$. Observe that $\psi\left(g, g^{\prime}\right) \geq 1$ and $\psi\left(g, g^{\prime}\right)=1$ when $g=g^{\prime}$. Our definition generalizes the concept of distortion between two metrics $g(p)$ and $g(q)$, as defined by Labelle and Shewchuk [17] (see Appendix B of the full version of this paper, [4]).

\subsection{Geodesy}

Let $v \in \mathbb{R}^{n}$. From the unique geodesic $\gamma$ satisfying $\gamma(0)=p$ with initial tangent vector $\dot{\gamma}=v$, one defines the exponential map through $\exp (v)=\gamma(1)$. The injectivity radius at a point $p$ of $\Omega$ is the largest radius for which the exponential map at $p$ restricted to a ball of that radius is a diffeomorphism. The injectivity radius $\iota_{\Omega}$ of $\Omega$ is defined as the infimum of the injectivity radii at all points. For any $p \in \Omega$ and for a two-dimensional linear subspace $H$ of the tangent space at $p$, we define the sectional curvature $K$ at $p$ for $H$ as the Gaussian curvature at $p$ of the surface $\exp _{p}(H)$.

In the theoretical studies of our algorithm, we will assume that the injectivity radius of $\Omega$ is strictly positive and its sectional curvatures are bounded.

\subsection{Power protected nets}

Controlling the quality of the Delaunay and Voronoi structures will be essential in our proofs. For this purpose, we use the notions of net and of power protection.

Power protection of point sets. Power protection of simplices is a concept formally introduced by Boissonnat, Dyer and Ghosh [2]. Let $\sigma$ be a simplex whose vertices belong to $\mathcal{P}$, and let $B_{g}(\sigma)=B_{g}(c, r)$ denote a circumscribing ball of $\sigma$ where $r=d_{g}(c, p)$ for any vertex $p$ of $\sigma$. We call $c$ the circumcenter of $\sigma$ and $r$ its circumradius.

For $0 \leq \delta \leq r$, we associate to $B_{g}(\sigma)$ the dilated ball $B_{g}^{+\delta}(\sigma)=B\left(c, \sqrt{r^{2}+\delta^{2}}\right)$. We say that $\sigma$ is $\delta$-power protected if $B_{g}^{+\delta}(\sigma)$ does not contain any point of $\mathcal{P} \backslash \operatorname{Vert}(\sigma)$ where $\operatorname{Vert}(\sigma)$ 
denotes the vertex set of $\sigma$. The ball $B_{g}^{+\delta}$ is the power protected ball of $\sigma$. Finally, a point set $\mathcal{P}$ is $\delta$-power protected if the Delaunay ball of its simplices are $\delta$-power protected.

Nets. To ensure that the simplices of the structures that we shall consider are well shaped, we will need to control the density and the sparsity of the point set. The concept of net conveys these requirements through sampling and separation parameters.

The sampling parameter is used to control the density of a point set: if $\Omega$ is a bounded domain, $\mathcal{P}$ is said to be an $\varepsilon$-sample set for $\Omega$ with respect to a metric field $g$ if $d_{g}(x, \mathcal{P})<\varepsilon$, for all $x \in \Omega$. The sparsity of a point set is controlled by the separation parameter: the set $\mathcal{P}$ is said to be $\mu$-separated with respect to a metric field $g$ if $d_{g}(p, q) \geq \mu$ for all $p, q \in \mathcal{P}$. If $\mathcal{P}$ is an $\varepsilon$-sample that is $\mu$-separated, we say that $\mathcal{P}$ is an $(\varepsilon, \mu)$-net.

\section{Riemannian Delaunay triangulations}

Given a metric field $g$, the Riemannian Voronoi diagram of a point set $\mathcal{P}$, denoted by $\operatorname{Vor}_{g}(\mathcal{P})$, is the Voronoi diagram built using the geodesic distance $d_{g}$. Formally, it is a partition of the domain in Riemannian Voronoi cells $\left\{V_{g}\left(p_{i}\right)\right\}$, where $\mathrm{V}_{g}\left(p_{i}\right)=\left\{x \in \Omega \mid d_{g}\left(p_{i}, x\right) \leq\right.$ $\left.d_{g}\left(p_{j}, x\right), \forall p_{j} \in \mathcal{P} \backslash p_{i}\right\}$.

The Riemannian Delaunay complex of $\mathcal{P}$ is an abstract simplicial complex, defined

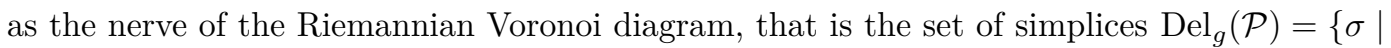
$\left.\operatorname{Vert}(\sigma) \in \mathcal{P}, \cap_{p \in \sigma} \mathrm{V}_{g}(p) \neq 0\right\}$. There is a straightforward duality between the diagram and the complex, and between their respective elements.

In this paper, we will consider both abstract simplices and complexes, as well as their geometric realization in $\mathbb{R}^{n}$ with vertex set $\mathcal{P}$. We now introduce two realizations of a simplex that will be useful, one curved and the other one straight.

The straight realization of a $n$-simplex $\sigma$ with vertices in $\mathcal{P}$ is the convex hull of its vertices. We denote it by $\bar{\sigma}$. In other words,

$$
\bar{\sigma}=\left\{x \in \Omega \subset \mathbb{R}^{n} \mid x=\sum_{p \in \sigma} \lambda_{p}(x) p, \lambda_{p}(x) \geq 0, \sum_{p \in \sigma} \lambda_{p}(x)=1\right\} .
$$

The curved realization, noted $\tilde{\sigma}$ is based on the notion of Riemannian center of mass [16, 13]. Let $y$ be a point of $\bar{\sigma}$ with barycentric coordinate $\lambda_{p}(y), p \in \sigma$. We can associate the energy functional $\mathcal{E}_{y}(x)=\frac{1}{2} \sum_{p \in \sigma} \lambda_{p}(y) d_{g}(x, p)^{2}$. We then define the curved realization of $\sigma$ as

$$
\tilde{\sigma}=\left\{\tilde{x} \in \Omega \subset \mathbb{R}^{n} \mid \tilde{x}=\operatorname{argmin} \mathcal{E}_{\bar{x}}(x), \bar{x} \in \bar{\sigma}\right\} .
$$

The edges of $\tilde{\sigma}$ are geodesic arcs between the vertices. Such a curved realization is well defined provided that the vertices of $\sigma$ lie in a sufficiently small ball according to the following theorem of Karcher [16].

- Theorem 1 (Karcher). Let the sectional curvatures $K$ of $\Omega$ be bounded, that is $\Lambda_{-} \leq K \leq$ $\Lambda_{+}$. Let us consider the function $\mathcal{E}_{y}$ on $B_{\rho}$, a geodesic ball of radius $\rho$ that contains the set $\left\{p_{i}\right\}$. Assume that $\rho \in \mathbb{R}^{+}$is less than half the injectivity radius and less than $\pi / 4 \sqrt{\Lambda_{+}}$ if $\Lambda_{+}>0$. Then $\mathcal{E}_{y}$ has a unique minimum point in $B_{\rho}$, which is called the center of mass.

Given an (abstract) simplicial complex $\mathcal{K}$ with vertices in $\mathcal{P}$, we define the straight (resp., curved) realization of $\mathcal{K}$ as the collection of straight (resp., curved) realizations of its simplices, and we write $\overline{\mathcal{K}}=\{\bar{\sigma}, \sigma \in \mathcal{K}\}$ and $\tilde{\mathcal{K}}=\{\tilde{\sigma}, \sigma \in \mathcal{K}\}$.

We will consider the case where $\mathcal{K}$ is $\operatorname{Del}_{g}(\mathcal{P})$. A simplex of $\overline{\operatorname{Del}}_{g}(\mathcal{P})$ will simply be called a straight Riemannian Delaunay simplex and a simplex of $\widetilde{\operatorname{Del}_{g}}(\mathcal{P})$ will be called a curved 
Riemannian Delaunay simplex, omitting "realization of". In the next two sections, we give sufficient conditions for $\overline{\operatorname{Del}}_{g}(\mathcal{P})$ and $\widetilde{\operatorname{Del}}_{g}(\mathcal{P})$ to be embedded in $\Omega$, in which case we will call them the straight and the curved Riemannian triangulations of $\mathcal{P}$.

\subsection{Sufficient conditions for $\widetilde{\operatorname{Del}_{g}}(\mathcal{P})$ to be a triangulation of $\mathcal{P}$}

It is known that $\widetilde{\operatorname{Del}_{g}}(\mathcal{P})$ is embedded in $\Omega$ under sufficient conditions. We give a short overview of these results. As in Dyer et al. [13], we define the non-degeneracy of a simplex $\tilde{\sigma}$ of $\widetilde{\operatorname{Del}}_{g}(\mathcal{P})$.

Definition 2. The curved realization $\tilde{\sigma}$ of a Riemannian Delaunay simplex $\sigma$ is said to be non-degenerate if and only if it is homeomorphic to the standard simplex.

Sufficient conditions for the complex $\widetilde{\operatorname{Del}}_{g}(\mathcal{P})$ to be embedded in $\Omega$ were given in [13]: a curved simplex is known to be non-degenerate if the Euclidean simplex obtained by lifting the vertices to the tangent space at one of the vertices via the exponential map has sufficient quality compared to the bounds on sectional curvature. Here, good quality means that the simplex is well shaped, which may be expressed either through its fatness (volume compared to longest edge length) or its thickness (smallest height compared to longest edge length).

Let us assume that, for each vertex $p$ of $\operatorname{Del}_{g}(\mathcal{P})$, all the curved Delaunay simplices in a neighborhood of $p$ are non-degenerate and patch together well. Under these conditions, $\widetilde{\operatorname{Del}}_{g}(\mathcal{P})$ is embedded in $\Omega$. We call $\widetilde{\operatorname{Del}_{g}}(\mathcal{P})$ the curved Riemannian Delaunay triangulation of $\mathcal{P}$.

\subsection{Sufficient conditions for $\overline{\operatorname{Del}}_{g}(\mathcal{P})$ to be a triangulation of $\mathcal{P}$}

Assuming that the conditions for $\widetilde{\operatorname{Del}_{g}}(\mathcal{P})$ to be embedded in $\Omega$ are satisfied, we now give conditions such that $\overline{\operatorname{Del}}_{g}(\mathcal{P})$ is also embedded in $\Omega$. The key ingredient will be a bound on the distance between a point of a simplex $\tilde{\sigma}$ and the corresponding point on the associated straight simplex $\bar{\sigma}$ (Lemma 3). This bound depends on the properties of the set of sites and on the local distortion of the metric field. When this bound is sufficiently small, $\overline{\mathrm{Del}}_{g}(\mathcal{P})$ is embedded in $\Omega$ as stated in Theorem 4 .

- Lemma 3. Let $\sigma$ be an $n$-simplex of $\operatorname{Del}_{g}(\mathcal{P})$. Let $\bar{x}$ be a point of $\bar{\sigma}$ and $\widetilde{x}$ the associated point on $\widetilde{\sigma}$ (as defined in Equation 1). If the geodesic distance $d_{g}$ is close to the Euclidean distance $d_{\mathbb{E}}$, i.e. the distortion $\psi\left(g, g_{\mathbb{E}}\right)$ is bounded by $\psi_{0}$, then $|\widetilde{x}-\bar{x}| \leq \sqrt{2 \cdot 4^{3}\left(\psi_{0}-1\right) \varepsilon^{2}}$.

We now apply Lemma 3 to the facets of the simplices of $\widetilde{\operatorname{Del}}_{g}(\mathcal{P})$. The altitude of the vertex $p$ in a simplex $\tau$ is noted $D(p, \tau)$.

- Theorem 4. Let $\mathcal{P}$ be a $\delta$-power protected $(\varepsilon, \mu)$-net with respect to $g$ on $\Omega$. Let $\sigma$ be any $n$-simplex of $\operatorname{Del}_{g}(\mathcal{P})$ and $p$ be any vertex of $\sigma$. Let $\tau$ be a facet of $\sigma$ opposite of vertex $p$. If, for all $\widetilde{x} \in \widetilde{\tau}$, we have $|\widetilde{x}-\bar{x}| \leq D\left(p_{i}, \sigma\right)$ ( $\bar{x}$ is defined in Equation 1$)$, then $\overline{\operatorname{Del}}_{d}(\mathcal{P})$ is embedded in $\Omega$.

The condition $|\widetilde{x}-\bar{x}| \leq D\left(p_{i}, \sigma\right)$ is achieved for a sufficiently dense sampling according to Lemma 3 and the fact that the distortion $\psi_{0}=\psi\left(g, g_{\mathbb{E}}\right)$ goes to 1 when the density increases. The complete proofs of Lemma 3 and Theorem 4 can be found in[4, Appendix F]. 


\section{Discrete Riemannian structures}

Although Riemannian Voronoi diagrams and Delaunay triangulations are appealing from a theoretical point of view, they are very difficult to compute in practice despite many studies [21]. To circumvent this difficulty, we introduce the discrete Riemannian Voronoi diagram. This discrete structure is easy to compute (see our companion paper [23] for details) and, as will be shown in the following sections, it is a good approximation of the exact Riemannian Voronoi diagram. In particular, their dual Delaunay structures are identical under appropriate conditions.

We assume that we are given a dense triangulation of the domain $\Omega$ we call the canvas and denote by $\mathcal{C}$. The canvas will be used to approximate geodesic distances between points of $\Omega$ and to construct the discrete Riemannian Voronoi diagram of $\mathcal{P}$. This bears some resemblance to the graph-induced complex of Dey et al. [11]. Notions related to the canvas will explicitly carry canvas in the name (for example, an edge of $\mathcal{C}$ is a canvas edge). In our analysis, we shall assume that the canvas is a dense triangulation, although weaker and more efficient structures can be used (see Section 9 and [23]).

\subsection{The discrete Riemannian Voronoi Diagram}

To define the discrete Riemannian Voronoi diagram of $\mathcal{P}$, we need to give a unique color to each site of $\mathcal{P}$ and to color the vertices of the canvas accordingly. Specifically, each canvas vertex is colored with the color of its closest site.

- Definition 5 (Discrete Riemannian Voronoi diagram). Given a metric field $g$, we associate to each site $p_{i}$ its discrete cell $\mathrm{V}_{g}^{\mathrm{d}}\left(p_{i}\right)$ defined as the union of all canvas simplices with at least one vertex of the color of $p_{i}$. We call the set of these cells the discrete Riemannian Voronoi diagram of $\mathcal{P}$, and denote it by $\operatorname{Vor}_{g}^{\mathrm{d}}(\mathcal{P})$.

Observe that contrary to typical Voronoi diagrams, our discrete Riemannian Voronoi diagram is not a partition of the canvas. Indeed, there is a one canvas simplex-thick overlapping since each canvas simplex $\sigma_{\mathcal{C}}$ belongs to all the Voronoi cells whose sites' colors appear in the vertices of $\sigma_{\mathcal{C}}$. This is intentional and allows for a straightforward definition of the complex induced by this diagram, as shown below.

\subsection{The discrete Riemannian Delaunay complex}

We define the discrete Riemannian Delaunay complex as the set of simplices $\operatorname{Del}_{g}^{\mathrm{d}}(\mathcal{P})=$ $\left\{\sigma \mid \operatorname{Vert}(\sigma) \in \mathcal{P}, \cap_{p \in \sigma} \mathrm{V}_{g}^{\mathrm{d}}(p) \neq 0\right\}$. Using a triangulation as canvas offers a very intuitive way to construct the discrete complex since each canvas $k$-simplex $\sigma$ of $\mathcal{C}$ has $k+1$ vertices $\left\{v_{0}, \ldots, v_{k}\right\}$ with respective colors $\left\{c_{0}, \ldots, c_{k}\right\}$ corresponding to the sites $\left\{p_{c_{0}}, \ldots, p_{c_{k}}\right\} \in \mathcal{P}$. Due to the way discrete Voronoi cells overlap, a canvas simplex $\sigma_{\mathcal{C}}$ belongs to each discrete Voronoi cell whose color appears in the vertices of $\sigma$. Therefore, the intersection of the discrete Voronoi cells $\left\{V_{g}^{\mathrm{d}}\left(p_{i}\right)\right\}$ whose colors appear in the vertices of $\sigma$ is non-empty and the simplex $\sigma$ with vertices $\left\{p_{i}\right\}$ thus belongs to the discrete Riemannian Delaunay complex. In that case, we say that the canvas simplex $\sigma_{\mathcal{C}}$ witnesses (or is a witness of) $\sigma$. For example, if the vertices of a canvas 3 -simplex $\tau_{\mathcal{C}}$ have colors yellow-blue-blue-yellow, then the intersection of the discrete Voronoi cells of the sites $p_{y e l l o w}$ and $p_{\text {blue }}$ is non-empty and the one-simplex $\sigma$ with vertices $p_{\text {yellow }}$ and $p_{\text {blue }}$ belongs to the discrete Riemannian Delaunay complex. The canvas simplex $\tau_{\mathcal{C}}$ thus witnesses the (abstract, for now) edge between $p_{\text {yellow }}$ and $p_{\text {blue }}$.

Figure 2 illustrates a canvas painted with discrete Voronoi cells, and the witnesses of the discrete Riemannian Delaunay complex. 


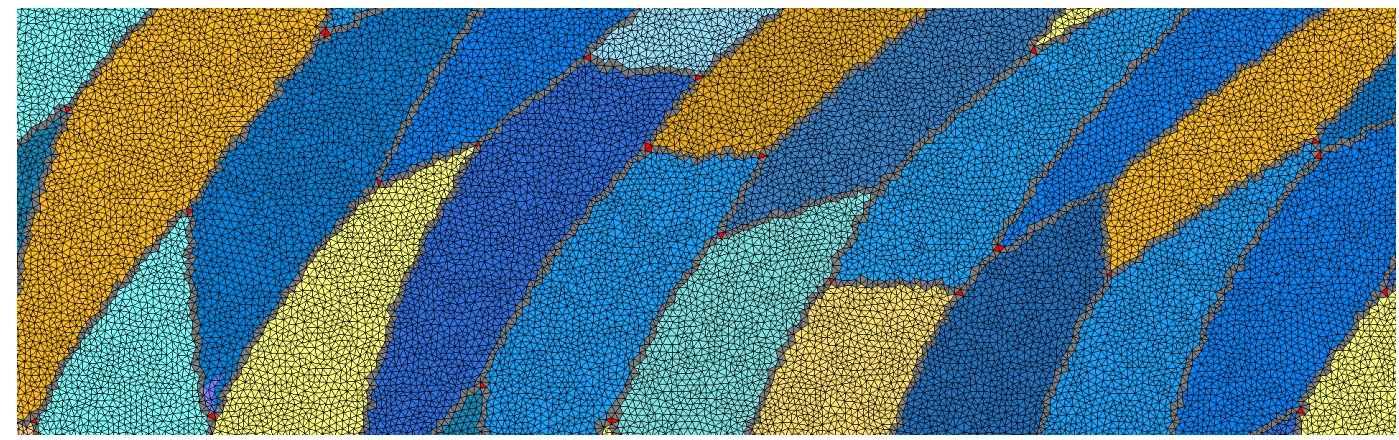

Figure 2 A canvas (black edges) and a discrete Riemannian Voronoi diagram drawn on it. The canvas simplices colored in red are witnesses of Voronoi vertices. The canvas simplices colored in grey are witnesses of Voronoi edges. Canvas simplices whose vertices all have the same color are colored with that color.

- Remark. If the intersection $\bigcap_{i=0 \ldots k} \mathrm{~V}_{g}^{\mathrm{d}}\left(p_{c_{i}}\right)$ is non-empty, then the intersection of any subset of $\left\{\mathrm{V}_{g}^{\mathrm{d}}\left(p_{c_{i}}\right)\right\}_{i=0 \ldots k}$ is non-empty. In other words, if a canvas simplex $\sigma_{\mathcal{C}}$ witnesses a simplex $\sigma$, then for each face $\tau$ of $\sigma$, there exists a face $\tau_{\mathcal{C}}$ of $\sigma_{\mathcal{C}}$ that witnesses $\tau$. As we assume that there is no boundary, the complex is pure and it is sufficient to only consider canvas $n$-simplices whose vertices have all different colors to build $\operatorname{Del}_{g}^{\mathrm{d}}(\mathcal{P})$.

Similarly to the definition of curved and straight Riemannian Delaunay complexes, we can define their discrete counterparts we respectively denote by $\widetilde{\operatorname{Del}_{g}^{\mathrm{d}}}(\mathcal{P})$ and $\overline{\operatorname{Del}}_{g}^{\mathrm{d}}(\mathcal{P})$. We will now exhibit conditions such that these complexes are well-defined and embedded in $\Omega$.

\section{Equivalence between the discrete and the exact structures}

We first give conditions such that $\operatorname{Vor}_{g}^{\mathrm{d}}(\mathcal{P})$ and $\operatorname{Vor}_{g}(\mathcal{P})$ have the same combinatorial structure, or, equivalently, that the dual Delaunay complexes $\operatorname{Del}_{g}(\mathcal{P})$ and $\operatorname{Del}_{g}^{\mathrm{d}}(\mathcal{P})$ are identical. Under these conditions, the fact that $\operatorname{Del}_{g}^{\mathrm{d}}(\mathcal{P})$ is embedded in $\Omega$ will immediately follow from the fact that the exact Riemannian Delaunay complex $\operatorname{Del}_{g}(\mathcal{P})$ is embedded (see Sections 3.1 and 3.2). It thus remains to exhibit conditions under which $\operatorname{Del}_{g}^{\mathrm{d}}(\mathcal{P})$ and $\operatorname{Del}_{g}(\mathcal{P})$ are identical.

Requirements will be needed on both the set of sites in terms of density, sparsity and protection, and on the density of the canvas. The central idea in our analysis is that power protection of $\mathcal{P}$ will imply a lower bound on the distance separating two non-adjacent Voronoi objects (and in particular two Voronoi vertices). From this lower bound, we will obtain an upper bound on the size on the cells of the canvas so that the combinatorial structure of the discrete diagram is the same as that of the exact one. The density of the canvas is expressed by $e_{\mathcal{C}}$, the length of its longest edge.

The main result of this paper is the following theorem.

- Theorem 6. Assume that $\mathcal{P}$ is a $\delta$-power protected $(\varepsilon, \mu)$-net in $\Omega$ with respect to $g$. Assume further that $\varepsilon$ is sufficiently small and $\delta$ is sufficiently large compared to the distortion between $g(p)$ and $g$ in an $\varepsilon$-neighborhood of $p$. Let $\left\{\lambda_{i}\right\}$ be the eigenvalues of $g(p)$ and $\ell_{0}$ a value that depends on $\varepsilon$ and $\delta$ (Precise bounds for $\varepsilon, \delta$ and $l_{0}$ are given in the proof). Then, if $e_{\mathcal{C}}<\min _{p \in \mathcal{P}}\left[\min _{i}\left(\sqrt{\lambda_{i}}\right) \min \left\{\mu / 3, \ell_{0} / 2\right\}\right], \operatorname{Del}_{g}^{d}(\mathcal{P})=\operatorname{Del}_{g}(\mathcal{P})$.

The rest of the paper will be devoted to the proof of this theorem. Our analysis is divided into two parts. We first consider in Section 6 the most basic case of a domain of $\mathbb{R}^{n}$ endowed 
with the Euclidean metric field. The result is given by Theorem 7 . The assumptions are then relaxed and we consider the case of an arbitrary metric field over $\Omega$ in Section 7. As we shall see, the Euclidean case already contains most of the difficulties that arise during the proof and the extension to more complex settings will be deduced from the Euclidean case by bounding the distortion.

\section{Equality of the Riemannian Delaunay complexes in the Euclidean setting}

In this section, we restrict ourselves to the case where the metric field is the Euclidean metric $g_{\mathbb{E}}$. To simplify matters, we initially assume that geodesic distances are computed exactly on the canvas. The following theorem gives sufficient conditions to have equality of the complexes.

Theorem 7. Assume that $\mathcal{P}$ is a $\delta$-power protected $(\varepsilon, \mu)$-net of $\Omega$ with respect to the Euclidean metric field $g_{\mathbb{E}}$. Denote by $\mathcal{C}$ the canvas, a triangulation with maximal edge length $e_{\mathcal{C}}$. If $e_{\mathcal{C}}<\min \left\{\mu / 16, \delta^{2} / 64 \varepsilon\right\}$, then $\operatorname{Del}_{\mathbb{E}}^{d}(\mathcal{P})=\operatorname{Del}_{\mathbb{E}}(\mathcal{P})$.

We shall now prove Theorem 7 by enforcing the two following conditions which, combined, give the equality between the discrete Riemannian Delaunay complex and the Riemannian Delaunay complex:

1. for every Voronoi vertex in the Riemannian Voronoi diagram $v=\cap_{\left\{p_{i}\right\}} V_{g}\left(p_{i}\right)$, there exists at least one canvas simplex with the corresponding colors $\left\{c_{p_{i}}\right\}$;

2. no canvas simplex witnesses a simplex that does not belong to the Riemannian Delaunay complex (equivalently, no canvas simplex has vertices whose colors are those of nonadjacent Riemannian Voronoi cells).

Condition 2 is a consequence of the separation of Voronoi objects, which in turn follows from power protection. The separation of Voronoi objects has previously been studied, for example by Boissonnat et al. [2]. Although the philosophy is the same, our setting is slightly more difficult and the results using power protection are new and use a more geometrical approach (see [4, Appendix C]).

\subsection{Sperner's Lemma}

Rephrasing Condition 1, we seek requirements on the density of the canvas $\mathcal{C}$ and on the nature of the point set $\mathcal{P}$ such that there exists at least one canvas $n$-simplex of $\mathcal{C}$ that has exactly the colors $c_{0}, \ldots, c_{d}$ of the vertices $p_{0}, \ldots, p_{d}$ of a simplex $\sigma$, for all $\sigma \in \operatorname{Del}_{g}(\mathcal{P})$. To prove the existence of such a canvas simplex, we employ Sperner's lemma [25], which is a discrete analog of Brouwer's fixed point theorem. We recall this result in Theorem 8 and illustrate it in a two-dimensional setting (inset).

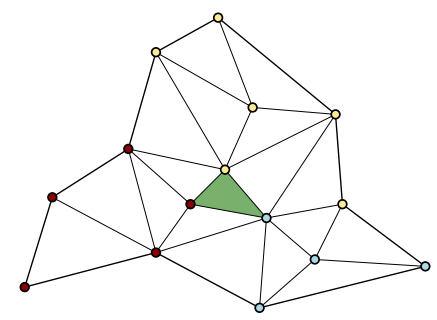




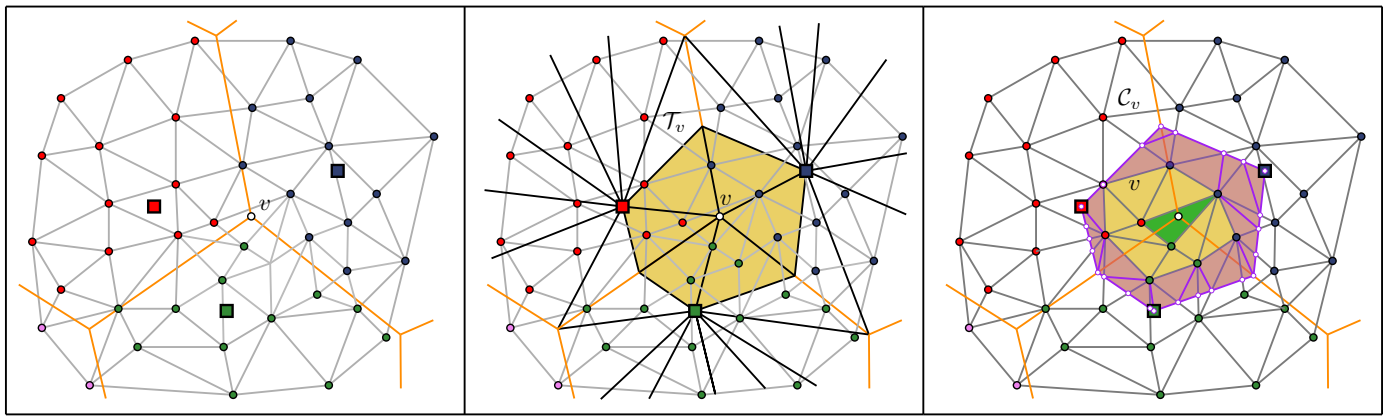

Figure 3 Illustration of the construction of $\mathcal{C}_{v}$. The Riemannian Voronoi diagram is drawn with thick orange edges and the sites are colored squares. The canvas is drawn with thin gray edges and colored circular vertices. The middle frame shows the subdivision of the incident Voronoi cells with think black edges and the triangulation $\mathcal{T}_{v}$ is drawn in yellow. On the right frame, the set of simplices $\mathcal{C}_{v}$ is colored in purple (simplices that do not belong to $\mathcal{C}$ ) and in dark yellow (simplices that belong to $\mathcal{C}$ ).

- Theorem 8 (Sperner's Lemma). Let $\sigma=\left(p_{0}, \ldots, p_{n}\right)$ be an $n$-simplex and let $T_{\sigma}$ denote a triangulation of the simplex. Let each vertex $v^{\prime} \in T_{\sigma}$ be colored such that the following conditions are satisfied:

- The vertices $p_{i}$ of $\sigma$ all have different colors.

- If a vertex $p^{\prime}$ lies on a $k$-face $\left(p_{i_{0}}, \ldots p_{i_{k}}\right)$ of $\sigma$, then $p^{\prime}$ has the same color as one of the vertices of the face, that is $p_{i_{j}}$.

Then, there exists an odd number of simplices in $T_{\sigma}$ whose vertices are colored with all $n+1$ colors. In particular, there must be at least one.

We shall apply Sperner's lemma to the canvas $\mathcal{C}$ and show that for every Voronoi vertex $v$ in the Riemannian Voronoi diagram, we can find a subset $\mathcal{C}_{v}$ of the canvas that fulfills the assumptions of Sperner's lemma, hence obtaining the existence of a canvas simplex in $\mathcal{C}_{v}$ (and therefore in $\mathcal{C}$ ) that witnesses $\sigma_{v}$. Concretely, the subset $\mathcal{C}_{v}$ is obtained in two steps:

- We first apply a barycentric subdivision of the Riemannian Voronoi cells incident to $v$. From the resulting set of simplices, we extract a triangulation $\mathcal{T}_{v}$ composed of the simplices incident to $v$ (Section 6.2).

- We then construct the subset $\mathcal{C}_{v}$ by overlaying the border of $\mathcal{T}_{v}$ and the canvas (Section 6.3). We then show that if the canvas simplices are small enough - in terms of edge length then $\mathcal{C}_{v}$ is the triangulation of a simplex that satisfies the assumptions of Sperner's lemma.

The construction of $\mathcal{C}_{v}$ is detailed in the following sections and illustrated in Figure 3: starting from a colored canvas (left), we subdivide the incident Voronoi cells of $v$ to obtain $\mathcal{T}_{v}$ (middle), and deduce the set of canvas simplices $\mathcal{C}_{v}$ which forms a triangulation that satisfies the hypotheses of Sperner's lemma, thus giving the existence of a canvas simplex (in green, right) that witnesses the Voronoi vertex within the union of the simplices, and therefore in the canvas.

\subsection{The triangulation $\mathcal{T}_{v}$}

For a given Voronoi vertex $v$ in the Euclidean Voronoi diagram $\operatorname{Vor}_{\mathbb{E}}(\mathcal{P})$ of the domain $\Omega$, the initial triangulation $\mathcal{T}_{v}$ is obtained by applying a combinatorial barycentric subdivision of the Voronoi cells of $\operatorname{Vor}_{\mathbb{E}}(\mathcal{P})$ that are incident to $v$ : to each Voronoi cell $V$ incident to $v$, we associate to each face $F$ of $V$ a point $c_{F}$ in $F$ which is not necessarily the geometric barycenter. We randomly associate to $c_{F}$ the color of any of the sites whose Voronoi cells intersect to 


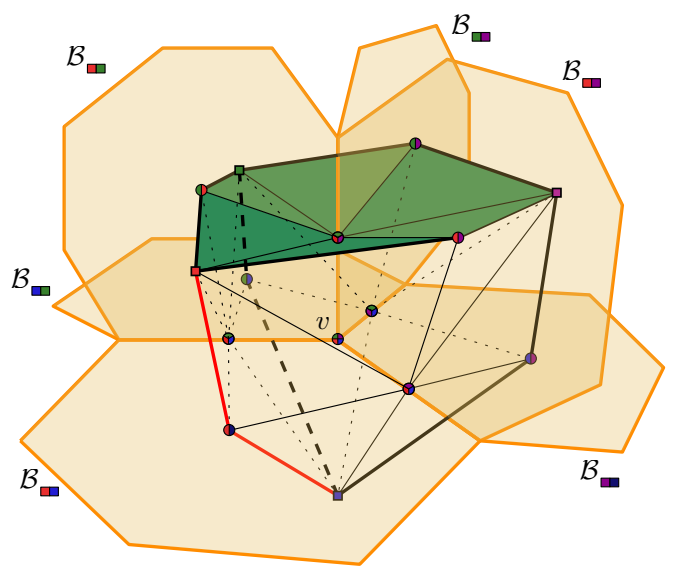

Figure 4 The triangulation $\mathcal{T}_{v}$ in 3D. A face (in green) and an edge (in red) of $\sigma_{\mathcal{S}}$.

give $F$. For example, in a two-dimensional setting, if the face $F$ is a Voronoi edge that is the intersection of $V_{\text {red }}$ and $V_{b l u e}$, then $c_{F}$ is colored either red or blue. Then, the subdivision of $V$ is computed by associating to all possible sequences of faces $\left\{F_{0}, F_{1}, \ldots F_{n-1}, F_{n}\right\}$ such that $F_{0} \subset F_{1} \cdots \subset F_{n}=V$ and $\operatorname{dim}\left(F_{i+1}\right)=\operatorname{dim}\left(F_{i}\right)+1$ the simplex with vertices $\left\{c_{F_{0}}, c_{F_{1}}, \ldots, c_{F_{n-1}}, c_{F_{n}}\right\}$. These barycentric subdivisions are allowed since Voronoi cells are convex polytopes.

Denote by $\Sigma_{V}$ the set of simplices obtained by barycentric subdivision of $V$ and $\Sigma_{v}=$ $\left\{\cup \Sigma_{V} \mid v \in V\right\}$. The triangulation $\mathcal{T}_{v}$ is defined as the star of $v$ in $\Sigma_{v}$, that is the set of simplices in $\Sigma_{v}$ that are incident to $v$. $\mathcal{T}_{v}$ is illustrated in Figure 4 in dimension 3. As shall be proven in Lemma $9, \mathcal{T}_{v}$ can be used to define a combinatorial simplex that satisfies the assumptions of Sperner's lemma.

\section{$\mathcal{T}_{v}$ as a triangulation of an $n$-simplex}

By construction, the triangulation $\mathcal{T}_{v}$ is a triangulation of the (Euclidean) Delaunay simplex $\sigma_{v}$ dual of $v$ as follows. We first perform the standard barycentric subdivision on this Delaunay simplex $\sigma_{v}$. We then map the barycenter of a $k$-face $\tau$ of $\sigma_{v}$ to the point $c_{F_{i}}$ on the Voronoi face $F_{i}$, where $F_{i}$ is the Voronoi dual of the $k$-face $\tau$. This gives a piecewise linear homeomorphism from the Delaunay simplex $\sigma_{v}$ to the triangulation $\mathcal{T}_{v}$. We call the image of this map the simplex $\sigma_{\mathcal{S}}$ and refer to the images of the faces of the Delaunay simplex as the faces of $\sigma_{\mathcal{S}}$. We can now apply Sperner's lemma.

- Lemma 9. Let $\mathcal{P}$ be a $\delta$-power protected $(\varepsilon, \mu)$-net. Let $v$ be a Voronoi vertex in the Euclidean Voronoi diagram, $\operatorname{Vor}_{\mathbb{E}}(\mathcal{P})$, and let $\Sigma_{v}$ be defined as above. The simplex $\sigma_{\mathcal{S}}$ and the triangulation $\mathcal{T}_{v}$ satisfy the assumptions of Sperner's lemma in dimension $n$.

Proof. By the piecewise linear map that we have described above, $\mathcal{T}_{v}$ is a triangulation of the simplex $\sigma_{\mathcal{S}}$. Because by construction the vertices $c_{F_{i}}$ lie on the Voronoi duals $F_{i}$ of the corresponding Delaunay face $\tau, c_{F_{i}}$ has the one of the colors of of the Delaunay vertices of $\tau$. Therefore, $\sigma_{\mathcal{S}}$ satisfies the assumptions of Sperner's lemma and there exists an $n$-simplex in $\mathcal{T}_{v}$ that witnesses $v$ and its corresponding simplex $\sigma_{v}$ in $\operatorname{Del}_{g}(\mathcal{P})$. 


\subsection{Building the triangulation $\mathcal{C}_{v}$}

Let $p_{i}$ be the vertices of the $k$-face $\tau_{\mathcal{S}}$ of $\sigma_{\mathcal{S}}$. In this section we shall assume not only that $\tau_{\mathcal{S}}$ is contained in the union of the Voronoi cells of $V\left(p_{i}\right)$, but in fact that $\tau_{\mathcal{S}}$ is a distance $8 e_{\mathcal{C}}$ removed from the boundary of $\cup V\left(p_{i}\right)$, where $e_{\mathcal{C}}$ is the longest edge length of a simplex in the canvas. We will now construct a triangulation $\mathcal{C}_{v}$ of $\sigma_{\mathcal{S}}$ such that:

- $\sigma_{\mathcal{S}}$ and its triangulation $\mathcal{C}_{v}$ satisfy the conditions of Sperner's lemma,

- the simplices of $\mathcal{C}_{v}$ that have no vertex that lies on the boundary $\partial \sigma_{\mathcal{S}}$ are simplices of the canvas $\mathcal{C}$.

The construction goes as follows. We first intersect the canvas $\mathcal{C}$ with $\sigma_{\mathcal{S}}$ and consider the canvas simplices $\sigma_{\mathcal{C}, i}$ such that the intersection of $\sigma_{\mathcal{S}}$ and $\sigma_{\mathcal{C}, i}$ is non-empty. These simplices $\sigma_{\mathcal{C}, i}$ can be subdivided into two sets, namely those that lie entirely in the interior of $\sigma_{\mathcal{S}}$, which we denote by $\sigma_{\mathcal{C}, i}^{\text {int }}$, and those that intersect the boundary, denoted by $\sigma_{\mathcal{C}, i}^{\partial}$.

The simplices $\sigma_{\mathcal{C}, i}^{\text {int }}$ are added to the set $\mathcal{C}_{v}$. We intersect the simplices $\sigma_{\mathcal{C}, i}^{\partial}$ with $\sigma_{\mathcal{S}}$ and triangulate the intersection. Note that $\sigma_{\mathcal{C}, i}^{\partial} \cap \sigma_{\mathcal{S}}$ is a convex polyhedron and thus triangulating it is not a difficult task. The vertices of the simplices in the triangulation of $\sigma_{\mathcal{C}, i}^{\partial} \cap \sigma_{\mathcal{S}}$ are colored according to which Voronoi cell they belong to. Finally, the simplices in the triangulation of $\sigma_{\mathcal{C}, i}^{\partial} \cap \sigma_{\mathcal{S}}$ are added to the set $\mathcal{C}_{v}$.

Since $\mathcal{T}_{v}$ is a triangulation of $\sigma_{\mathcal{S}}$, the set $\mathcal{C}_{v}$ is by construction also a triangulation of $\sigma_{\mathcal{S}}$. This triangulation trivially gives a triangulation of the faces $\tau_{\mathcal{S}}$. Because we assume that $\tau_{\mathcal{S}}$ is contained in the union of its Voronoi cells, with a margin of $8 e_{\mathcal{C}}$ we now can draw two important conclusions:

- The vertices of the triangulation of each face $\tau_{\mathcal{S}}$ have the colors of the vertices $p_{i}$ of $\tau_{\mathcal{S}}$.

- None of the simplices in the triangulation of $\sigma_{\mathcal{C}, i}^{\partial} \cap \sigma_{\mathcal{S}}$ can have $n+1$ colors, because every such simplex must be close to one face $\tau_{\mathcal{S}}$, which means that it must be contained in the union of the Voronoi cells $V\left(p_{i}\right)$ of the vertices of $\tau_{\mathcal{S}}$.

We can now invoke Sperner's lemma; $\mathcal{C}_{v}$ is a triangulation of the simplex $\sigma_{\mathcal{S}}$ whose every face has been colored with the appropriate colors (since $\sigma_{\mathcal{S}}$ triangulated by $\mathcal{T}_{v}$ satisfies the assumptions of Sperner's lemma, see Lemma 9). This means that there is a simplex $\mathcal{C}_{v}$ that is colored with $n+1$ colors. Because of our second observation above, the simplex with these $n+1$ colors must lie in the interior of $\sigma_{\mathcal{S}}$ and is thus a canvas simplex.

We summarize by the following lemma:

- Lemma 10. If every face $\tau_{\mathcal{S}}$ of $\sigma_{\mathcal{S}}$ with vertices $p_{i}$ is at distance $8 e_{\mathcal{C}}$ from the boundary of the union of its Voronoi cells $\partial\left(\cup V\left(p_{i}\right)\right)$, then there exists a canvas simplex in $\mathcal{C}_{v}$ such that it is colored with the same vertices as the vertices of $\sigma_{\mathcal{S}}$.

The key task that we now face is to guarantee that faces $\tau_{\mathcal{S}}$ indeed lie well inside of the union of the appropriate Voronoi regions. This requires first and foremost power protection. Indeed, if a point set is power protected, the distance between a Voronoi vertex $c$ and the Voronoi faces that are not incident to $c$, which we will refer to from now on as foreign Voronoi faces, can be bounded, as shown in the following Lemma:

- Lemma 11. Suppose that $c$ is the circumcenter of a $\delta$-power protected simplex $\sigma$ of a Delaunay triangulation built from an $\varepsilon$-sample, then all foreign Voronoi faces are at least $\delta^{2} / 8 \varepsilon$ far from $c$.

The proof of this Lemma is given in the full version of this paper (see [4, Section C.2]).

In almost all cases, this result gives us the distance bound we require: we can assume that vertices $\left\{c_{F_{0}}, c_{F_{1}}, \ldots, c_{F_{n-1}}, c_{F_{n}}\right\}$ which we used to construct $\mathcal{T}_{v}$, are well placed, meaning 

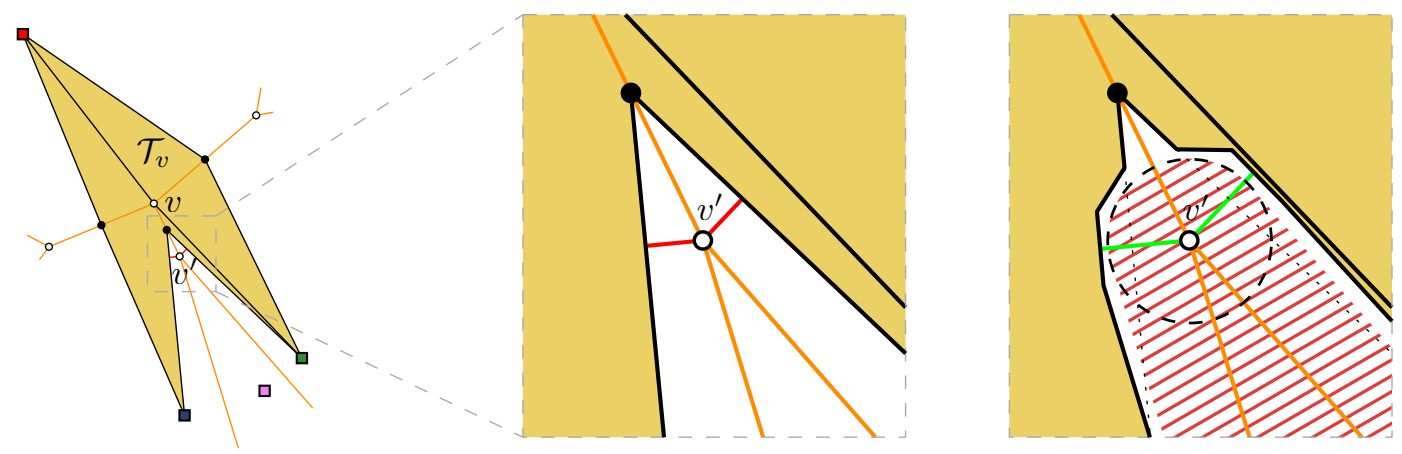

Figure 5 The point $v^{\prime}$ can be arbitrarily close to $\mathcal{T}_{v}$, as shown by the red segments (left and center). After piecewise linear deformation, this issue is resolved, as seen by the green segments (right).

that there is a minimum distance between these vertices and foreign Voronoi objects. However it can still occur that foreign Voronoi objects are close to a face $\tau_{\mathcal{S}}$ of $\sigma_{\mathcal{S}}$. This occurs even in two dimensions, where a Voronoi vertex $v^{\prime}$ can be very close to a face $\tau_{\mathcal{S}}$ because of obtuse angles, as illustrated in Figure 5.

Thanks to power protection, we know that $v^{\prime}$ is removed from foreign Voronoi objects. This means that we can deform $\sigma_{\mathcal{S}}$ (in a piecewise linear manner) in a neighborhood of $v^{\prime}$ such that the distance between $v^{\prime}$ and all the faces of the deformed $\sigma_{\mathcal{S}}$ is lower bounded.

In general the deformation of $\sigma_{\mathcal{S}}$ is performed by "radially pushing" simplices away from the foreign Voronoi faces of $v$ with a ball of radius $r=\min \left\{\mu / 16, \delta^{2} / 64 \varepsilon\right\}$. The value $\mu / 16$ is chosen so that we do not move any vertex of $\sigma_{v}$ (the dual of $v$ ): indeed, $\mathcal{P}$ is $\mu$-separated and thus $d_{\mathbb{E}}\left(p_{i}, p_{j}\right)>\mu$. The value $\delta^{2} / 64 \varepsilon$ is chosen so that $\sigma_{\mathcal{S}}$ and its deformation stay isotopic (no "pinching" can happen), using Lemma 11. In fact it is advisable to use a piecewise linear version of "radial pushing", to ensure that the deformation of $\sigma_{\mathcal{S}}$ is a polyhedron. This guarantees that we can triangulate the intersection, see Chapter 2 of Rourke and Sanderson [22]. After this deformation we can follow the steps we have given above to arrive at a well-colored simplex.

- Lemma 12. Let $\mathcal{P}$ be a $\delta$-power protected $(\varepsilon, \mu)$-net. Let $v$ be a Voronoi vertex of the Euclidean Voronoi diagram $\operatorname{Vor}_{\mathbb{E}}(\mathcal{P})$, and $\mathcal{T}_{v}$ as defined above. If the length $e_{\mathcal{C}}$ of the longest canvas edge is bounded as follows: $e_{\mathcal{C}}<r=\min \left\{\mu / 16, \delta^{2} / 64 \varepsilon\right\}$, then there exists a canvas simplex that witnesses $v$ and the corresponding simplex $\sigma_{v}$ in $\operatorname{Del}_{\mathbb{E}}(\mathcal{P})$.

\section{Conclusion}

So far, we have only proven that $\operatorname{Del}_{g}(\mathcal{P}) \subseteq \operatorname{Del}_{g}^{\mathrm{d}}(\mathcal{P})$. The other inclusion, which corresponds to Condition 2 mentioned above, is much simpler: as long as a canvas edge is shorter than the smallest distance between a Voronoi vertex and a foreign face of the Riemannian Voronoi diagram, then no canvas simplex can witness a simplex that is not in $\operatorname{Del}_{g}(\mathcal{P})$. Such a bound is already given by Lemma 11 and thus, if $e_{\mathcal{C}}<\delta^{2} / 8 \varepsilon$ then $\operatorname{Del}_{g}^{\mathrm{d}}(\mathcal{P}) \subseteq \operatorname{Del}_{g}(\mathcal{P})$. Observe that this requirement is weaker than the condition imposed in Lemma 12 and it was thus already satisfied. It follows that $\operatorname{Del}_{g}^{\mathrm{d}}(\mathcal{P})=\operatorname{Del}_{g}(\mathcal{P})$ if $e_{\mathcal{C}}<\min \left\{\mu / 16, \delta^{2} / 64 \varepsilon\right\}$, which concludes the proof of Theorem 7 .

- Remark. Assuming that the point set is a $\delta$-power protected $(\varepsilon, \mu)$-net might seem like a strong assumption. However, it should be observed that any non-degenerate point set can be 
seen as a $\delta$-power protected $(\varepsilon, \mu)$-net, for a sufficiently large value of $\varepsilon$ and sufficiently small values of $\delta$ and $\mu$. Our results are therefore always applicable but the necessary canvas density increases as the quality of the point set worsens (Lemma 12). In our practical companion paper [23, Section 7], we showed how to generate $\delta$-power protected $(\varepsilon, \mu)$-nets for given values of $\varepsilon, \mu$ and $\delta$.

\section{Extension to more complex settings}

In the previous section, we have placed ourselves in the setting of an (open) domain endowed with the Euclidean metric field. To prove Theorem 6, we need to generalize Theorem 7 to more general metrics, which will be done in the two following subsections.

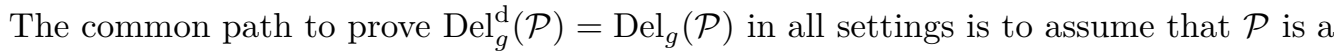
power protected net with respect to the metric field. We then use the stability of entities under small metric perturbations to take us back to the now solved case of the domain $\Omega$ endowed with an Euclidean metric field. Separation and stability of Delaunay and Voronoi objects has previously been studied by Boissonnat et al. [2, 3], but our work lives in a slightly more complicated setting. Moreover, our proofs are generally more geometrical and sometimes simpler. For completeness, the extensions of these results to our context are detailed in the full version of this paper [4, Appendices $\mathrm{C}$ and $\mathrm{E}]$.

We now detail the different intermediary settings. For completeness, the full proofs are included in the appendices.

\subsection{Uniform metric field}

We first consider the rather easy case of a non-Euclidean but uniform (constant) metric field over an (open) domain. The square root of a metric gives a linear transformation between the base space where distances are considered in the metric and a metric space where the Euclidean distance is used (see [4, Appendix B.1]). Additionally, we show that a $\delta$-power protected $(\varepsilon, \mu)$-net with respect to the uniform metric is, after transformation, still a $\delta$-power protected $(\varepsilon, \mu)$-net but with respect to the Euclidean setting [4, Lemma 26], bringing us back to the setting we have solved in Section 6. Bounds on the power protection, sampling and separation coefficients, and on the canvas edge length can then be obtained from the result for the Euclidean setting, using Theorem 12. These bounds can be transported back to the case of uniform metric fields by scaling these values according to the smallest eigenvalue of the metric [4, Theorem 40].

\subsection{Arbitrary metric field}

The case of an arbitrary metric field over $\Omega$ is handled by observing that an arbitrary metric field is locally well-approximated by a uniform metric field. It is then a matter of controlling the distortion.

We first show that, for any point $p \in \Omega$, density separation and power protection are locally preserved in a neighborhood $U_{p}$ around $p$ when the metric field $g$ is approximated by the constant metric field $g^{\prime}=g(p)$ [4, Lemmas 27 and 39]: if $\mathcal{P}$ is a $\delta$-power protected $(\varepsilon, \mu)$-net with respect to $g$, then $\mathcal{P}$ is a $\delta^{\prime}$-power protected $\left(\varepsilon^{\prime}, \mu^{\prime}\right)$-net with respect to $g^{\prime}$. Previous results can now be applied to obtain conditions on $\delta^{\prime}, \varepsilon^{\prime}, \mu^{\prime}$ and on the (local) maximal length of the canvas such that $\operatorname{Del}_{g}^{\mathrm{d}}(\mathcal{P})=\operatorname{Del}_{g}(\mathcal{P})$ (see $[4$, Lemma 41]).

These local triangulations can then be stitched together to form a triangulation embedded in $\Omega$. The (global) bound on the maximal canvas edge length is given by the minimum of 
the local bounds, each computed through the results of the previous sections. This ends the proof of Theorem 6 .

Once the equality between the complexes is obtained, conditions giving the embeddability of the discrete Karcher Delaunay triangulation and the discrete straight Delaunay triangulation are given by previous results that we have established in Sections 3.1 and 3.2 respectively.

\section{Extensions of the main result}

Approximate geodesic computations. Approximate geodesic distance computations can be incorporated in the analysis of the previous section by observing that computing inaccurately geodesic distances in a domain $\Omega$ endowed with a metric field $g$ can be seen as computing exactly geodesic distances in $\Omega$ with respect to a metric field $g^{\prime}$ that is close to $g$ [4, Section H.3].

General manifolds. The previous section may also be generalized to an arbitrary smooth $n$-manifold $\mathcal{M}$ embedded in $\mathbb{R}^{m}$. We shall assume that, apart from the metric induced by the embedding of the domain in Euclidean space, there is a second metric $g$ defined on $\mathcal{M}$. Let $\pi_{p}: \mathcal{M} \rightarrow T_{p} \mathcal{M}$ be the orthogonal projection of points of $\mathcal{M}$ on the tangent space $T_{p} \mathcal{M}$ at $p$. For a sufficiently small neighborhood $U_{p} \subset T_{p} \mathcal{M}, \pi_{p}$ is a local diffeomorphism (see Niyogi [20]).

Denote by $\mathcal{P}_{T_{p}}$ the point set $\left\{\pi_{p}\left(p_{i}\right), p_{i} \in \mathcal{P}\right\}$ and $\mathcal{P}_{U_{p}}$ the restriction of $\mathcal{P}_{T_{p}}$ to $U_{p}$. Assuming that the conditions of Niyogi et al. [20] are satisfied (which are simple density constraints on $\varepsilon$ compared to the reach of the manifold), the pullback of the metric with the inverse projection $\left(\pi_{p}^{-1}\right)^{*} g$ defines a metric $g_{p}$ on $U_{p}$ such that for all $q, r \in U_{p}, d_{g_{p}}(q, r)=$ $d_{g}\left(\pi_{p}^{-1}(q), \pi_{p}^{-1}(r)\right)$. This implies immediately that if $\mathcal{P}$ is a $\delta$-power protected $(\varepsilon, \mu)$-net on $\mathcal{M}$ with respect to $g$ then $\mathcal{P}_{U_{p}}$ is a $\delta$-power protected $(\varepsilon, \mu)$-net on $U_{p}$. We have thus a metric on a subset of a $n$-dimensional space, in this case the tangent space, giving us a setting that we have already solved. It is left to translate the sizing field requirement from the tangent plane to the manifold $\mathcal{M}$ itself. Note that the transformation $\pi_{p}$ is completely independent of $g$. Boissonnat et al. [2, Lemma 3.7] give bounds on the metric distortion of the projection on the tangent space. This result allows to carry the canvas sizing field requirement from the tangent space to $\mathcal{M}$.

\section{Implementation}

The construction of the discrete Riemannian Voronoi diagram and of the discrete Riemannian Delaunay complex has been implemented for $n=2,3$ and for surfaces of $\mathbb{R}^{3}$. An in-depth description of our structure and its construction as well as an empirical study can be found in our practical paper [23]. We simply make a few observations here.

The theoretical bounds on the canvas edge length provided by Theorems 6 and 7 are far from tight and thankfully do not need to be honored in practice. A canvas whose edge length are about a tenth of the distance between two seeds suffices. This creates nevertheless unnecessarily dense canvasses since the density does not in fact need to be equal everywhere at all points and even in all directions. This issue is resolved by the use of anisotropic canvasses.

Our analysis was based on the assumption that all canvas vertices are painted with the color of the closest site. In our implementation, we color the canvas using a multiple-front 
vector Dijkstra algorithm [5], which empirically does not suffer from the same convergence issues as the traditional Dijkstra algorithm, starting from all the sites. It should be noted that any geodesic distance computation method can be used, as long as it converges to the exact geodesic distance when the canvas becomes denser. The Riemannian Delaunay complex is built on the fly during the construction of the discrete Riemannian Voronoi diagram: when a canvas simplex is first fully colored, its combinatorial information is extracted and the corresponding simplex is added to $\operatorname{Del}_{g}(\mathcal{P})$.

Acknowledgments. We thank Ramsay Dyer for enlightening discussions.

\section{References}

1 F. Aurenhammer and R. Klein. Voronoi diagrams. In J. Sack and G. Urrutia, editors, Handbook of Computational Geometry, pages 201-290. Elsevier Science Publishing, 2000.

2 J.-D. Boissonnat, R. Dyer, and A. Ghosh. Delaunay triangulation of manifolds. Foundations of Computational Mathematics, pages 1-33, 2017.

3 J.-D. Boissonnat, R. Dyer, A. Ghosh, and S. Y. Oudot. Only distances are required to reconstruct submanifolds. Comp. Geom. Theory and Appl., 2016. To appear.

4 J.-D. Boissonnat, M. Rouxel-Labbé, and M. Wintraecken. Anisotropic triangulations via discrete Riemannian Voronoi diagrams, 2017. URL: https://arxiv.org/abs/1703. 06487.

5 M. Campen, M. Heistermann, and L. Kobbelt. Practical anisotropic geodesy. In Proceedings of the Eleventh Eurographics/ACMSIGGRAPH Symposium on Geometry Processing, SGP'13, pages 63-71. Eurographics Association, 2013.

6 G. D. Cañas and S. J. Gortler. Orphan-free anisotropic Voronoi diagrams. Discrete and Computational Geometry, 46(3), 2011.

7 G. D. Cañas and S.J. Gortler. Duals of orphan-free anisotropic Voronoi diagrams are embedded meshes. In SoCG, pages 219-228. ACM, 2012.

8 T. Cao, H. Edelsbrunner, and T. Tan. Proof of correctness of the digital Delaunay triangulation algorithm. Comp. Geo.: Theory and Applications, 48, 2015.

9 S.-W. Cheng, T. K. Dey, E. A. Ramos, and R. Wenger. Anisotropic surface meshing. In Proceedings of the Seventeenth Annual ACM-SIAM Symposium on Discrete Algorithms, pages 202-211. Society for Industrial and Applied Mathematics, 2006.

10 E. F. D'Azevedo and R. B. Simpson. On optimal interpolation triangle incidences. SIAM J. Sci. Statist. Comput., 10(6):1063-1075, 1989.

11 T. K. Dey, F. Fan, and Y. Wang. Graph induced complex on point data. Computational Geometry, 48(8):575-588, 2015.

12 Q. Du and D. Wang. Anisotropic centroidal Voronoi tessellations and their applications. SIAM Journal on Scientific Computing, 26(3):737-761, 2005.

13 R. Dyer, G. Vegter, and M. Wintraecken. Riemannian simplices and triangulations. Preprint: arXiv:1406.3740, 2014.

14 R. Dyer, H. Zhang, and T. Möller. Surface sampling and the intrinsic Voronoi diagram. Computer Graphics Forum, 27(5):1393-1402, 2008.

15 M. Garland and P. S. Heckbert. Surface simplification using quadric error metrics. In $A C M$ SIGGRAPH, pages 209-216, 1997.

16 H. Karcher. Riemannian center of mass and mollifier smoothing. Communications on Pure and Applied Mathematics, 30:509-541, 1977.

17 F. Labelle and J.R. Shewchuk. Anisotropic Voronoi diagrams and guaranteed-quality anisotropic mesh generation. In SCG'03: Proceedings of the Nineteenth Annual Symposium on Computational Geometry, pages 191-200. ACM, 2003. 
18 G. Leibon. Random Delaunay triangulations, the Thurston-Andreev theorem, and metric uniformization. PhD thesis, UCSD, 1999.

19 J.-M. Mirebeau. Optimal meshes for finite elements of arbitrary order. Constructive approximation, 32(2):339-383, 2010.

20 P. Niyogi, S. Smale, and S. Weinberger. Finding the homology of submanifolds with high confidence from random samples. Discrete \& Comp. Geom., 39(1-3), 2008.

21 G. Peyré, M. Péchaud, R. Keriven, and L. D. Cohen. Geodesic methods in computer vision and graphics. Found. Trends. Comput. Graph. Vis., 2010.

22 C. Rourke and B. Sanderson. Introduction to piecewise-linear topology. Springer Science \& Business Media, 2012.

23 M. Rouxel-Labbé, M. Wintraecken, and J.-D. Boissonnat. Discretized Riemannian Delaunay triangulations. In Proc. of the 25th Intern. Mesh. Round. Elsevier, 2016.

24 J.R. Shewchuk. What is a good linear finite element? Interpolation, conditioning, anisotropy, and quality measures, Manuscript 2002.

25 E. Sperner. Fifty years of further development of a combinatorial lemma. Numerical solution of highly nonlinear problems, pages 183-197, 1980. 